\title{
SYSTEMIC LUPUS ERYTHEMATOSUS TREATMENT IN PREGNANCY: CASE STUDY
}

\author{
Laucekova $Z^{1}$, Biskupska Bodova $K^{1}$ Nachajova $M^{1}$, Kudela $E^{1}$, Fiolka $R^{1}$, Pridavkova $D^{2}$, \\ STASKOVA $\mathrm{K}^{2}$, BIRINGER K. ${ }^{1}$
}
${ }^{1}$ Clinic of Gynecology and Obstetrics, Jessenius Faculty of Medicine in Martin, Comenius University in Bratislava, and Martin University Hospital, Martin, Slovak Republic
${ }^{2}$ Clinic of Internal Medicine I., Jessenius Faculty of Medicine in Martin, Comenius University in Bratislava, and Martin University Hospital, Martin, Slovak Republic

\begin{abstract}
A b s tract
Systemic lupus erythematosus is a chronic inflammatory autoimmune disease with high prevalence in female in reproductive age. In recent years the prognosis of pregnant patients with SLE has improved significantly. Even though the treatment options have improved, the risk of flares, preeclampsia, pregnancy loss, and premature labours remains high compared to healthy women. The aim of this article is to offer a review of current treatment options in pregnant patients with SLE and to present a case report of 32-year-old patient with newly diagnosed acute outbreak of SLE, who experienced a life-threatening multisystem flare at 24 weeks of gestational age. This case represents one of the most extreme manifestations of lupus disease activity associated with pregnancy that has been reported in literature and emphasizes the importance of preconception evaluation and counseling and amultidisciplinary management approach in cases with a complex and evolving clinical course.
\end{abstract}

Key words: Systemic lupus erythematosus, treatment, plasmapheresis, imunoabsorption

\section{INTRODUCTION}

Systemic lupus erythematosus is a complex autoimmune disease with various clinical manifestations and disease course. With introduction of combined glucocorticoid and immunosuppressive therapy the prognosis of patients with SLE has improved significantly (1). However, there is still a group of patients who do not respond to standard therapy and their disease is classified as refractory lupus. These patients remain a therapeutic challenge. The definitions of the refractory lupus are various: in the case of nephritic lupus they may include gradual deterioration of renal function, persistent nephrotic syndrome, and inability to achieve a partial response in proteinuria within 12 months or a complete response within 24 months (2). However, these definitions do not apply to other serious or life-threatening forms of the disease, such as refractory skin, neuropsychiatric or hematological SLE. Current recommendations recommend other glucocorticoids, alternation of immunosuppressive drugs such as mycophenolate mofetil, cyclophosphamide, and rituximab, as well as consideration of alternative forms of treatment that include plasmapheresis/ plasma exchange (PLEX), immunoadsorption (IAS), or intravenous imunoglobuline therapy. Many of the therapeutic modalities are contraindicated in pregnancy and the treatment of refractory forms of the disease during pregnancy remains a challenge (2-3).

Corresponding author: Zuzana Laučeková, M.D; e-mail: zuzana_laucekova@yahoo.com

(C) 2020 Zuzana Laucekova et al.

This work is licensed under the Creative Commons Attribution-NonCommercial-NoDerivs 4.0 License (https://creativecommons.org/licenses/by-nc-nd/4.0/) 


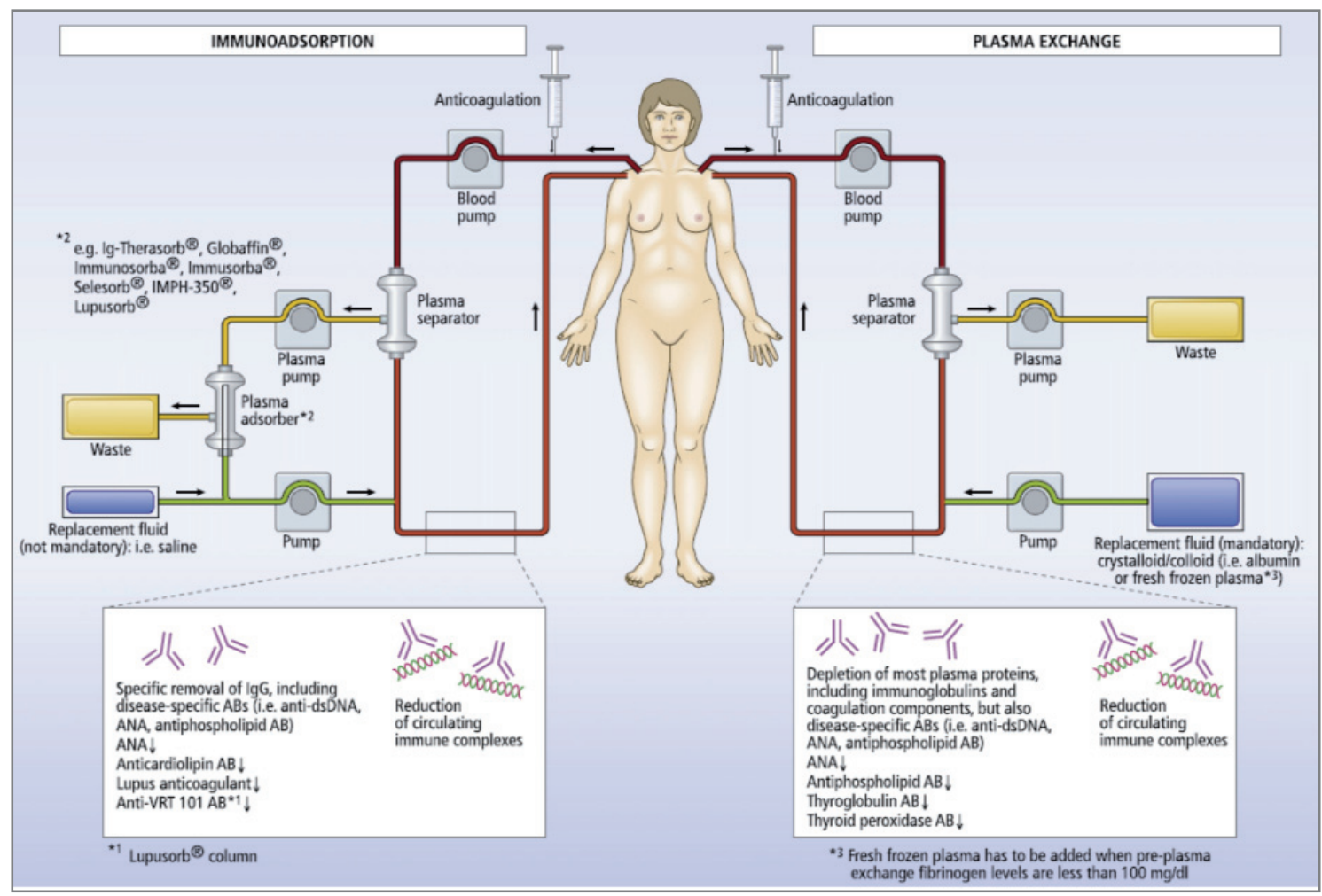

Fig. 1 Technical aspects and molecular changes exerted by immunoadsorption (left side) and plasma exchange (right side) in systemic lupus erythematosus. (2)

\section{CASE PRESENTATION}

A 32-year-old patient was admitted to our ward at the 24th week of pregnancy with a newly diagnosed acute outbreak of SLE. After uncomplicated first pregnancy the patient underwent two spontaneous abortions in the first trimester. Hematologic examination for repeated abortions confirmed a thrombophilic status of PAI4G / 5G and PAI-I, SPS and slight FIX deficiency (43\%). Due to her diagnosis she was recommended to take ASA 100mg daily (21). In the 10th week of pregnancy the incidence of skin rash, alopecia, and seborrhea were reported. The patient was followed by a dermatovenerologist and was given topical therapy without improvement. From the 22nd week of pregnancy the patient was hospitalized at the gynecology-obstetric ward at a regional hospital for aggravation of large joints pain, hematuria, and recurrent severe epistaxis leading to moderately severe anemia. During this hospital stay SLE developed and progressed into multiorgan symptomatics. Hematological, internal, nephrological, and neurological consultations were performed. Given the severity of the general health condition the patient was transferred to the perinatological center at the 24 th week of pregnancy.

Initial gynecological examination confirmed fetal normotrophy, adequate amount of amniotic fluid, and fetus without developmental malformations. Due to the severity of the condition the patient was transferred to the metabolic ICU of an internal clinic where she was monitored for hemodynamically severe gastrointestinal bleeding. Initial examinations revealed severe involvement of several organ systems - nephrotic syndrome with moderate proteinuria and peripheral edema associated with lupus nephritis, cutaneous erythema of 
the whole body, vasculitis with acute necrosis of the last articles of the fingers and toes (Fig. 2), mucosal ulcerations of the mouth, the gastrointestinal ulcer and laboratory-confirmed severe grade thrombocytopenia, severe grade normochromic normocytic anemia very likely post-haemorrhagic in combination with primary disease (positive direct Coombs test, low serum haptoglobin levels) and lymphopenia, high autoantibody titers ANA, positive anti-histone anti-dsDNA and anti-SSA / Ro antibodies, hypocomplementemia C3 and C4, mono-isotype hypogammaglobulinemia of mild degree while other Ig isotypes were normal, hypoproteinaemia, hypoalbuminaemia, hypokalaemia, and hypomagnesaemia. Antiphospholipid antibodies were not present.

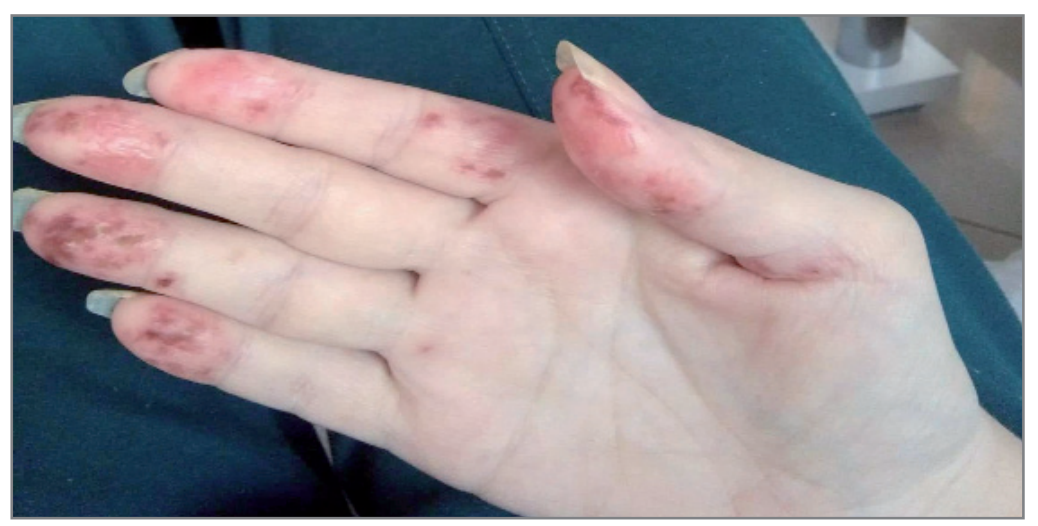

Fig. 2 Vasculitis with acute necrosis of the last articles of the fingers

In the beginning of the hospitalization the patient was informed about the severity of her health state and the risks associated with treatment and continued pregnancy. Due to the desired pregnancy and low gestational week of the fetus the patient asked to continue with the pregnancy. Pulse treatment with methylprednisone (250mg per 24h) was initiated followed by a switch to continued HCN corticotherapy. HCQ (200mg per 24h) was added to the therapy. Due to severe grade normochromic normocytic anemia most likely post-haemorrhagic patient ounderwent repeated haemosubstitution. Pantoprazole, Dicynone, Pamba, folic acid, vitamin K, vitamin D, substitution of calcium and other minerals and protein food additives were gradually added to the therapy.

Replacement PLEX was then added to the pharmacological treatment. A total of 10 cycles of PLEX were performed within 14 days. Despite clinically improved health the high disease activity with high antibody titres persisted. For persistent hypoalbuminaemia with lower limbs edema, intravenous albumin was administered. Upon completion of 10 cycles of PLEX, partial clinical and laboratory improvement followed and the rheumatologist recommended switching from intravenous HCN therapy to oral dexamethasone therapy. Due to the development of complex immunodeficiency state including humoral immunity, complex immune dysregulation, gradual progression of moderate thrombocytopenia, as well as present anemia and lymphopenia (Evans syndrome), borderline hypogammaglobulinaemia of IgG, as well as high risk for fetus, imunologist recommended intravenous immunoglobulin therapy ( 60 grams were administrated in total within 3 days). During the hospital stay the health status was complicated by fevers and increased inflammatory activity. Klebsiella pneumoniae and Escherichia Coli infection were present in urine and blood cultures. Depending on sensitivity the patient was treated with intravenous cefotaxime with good effect. For the possible development of endocarditis an echocardiographic examination was performed with the finding of dilated left atrium $(45 \mathrm{~mm})$, significant pulmonary hypertension (without right-ventricular dysfunction) but without the presence of vegetation on the valves. After 4 weeks a repeated immunoglobulin therapy at 33 weeks of gestation was administrated, again without adverse effects. 
During her stay at the Clinic of Internal Medicine internal clinic the patient underwent regular fetal ultrasound gynecological examinations at weekly intervals. At the $27^{\text {th }}$ gestational week a fetal echocardiography was performed to exclude the fetal congenital heart block with a negative finding in means of gross developmental defects. From the 30th week of pregnancy a restriction of fetal growth was observed with preserved normohydramnion and no signs of fetal centralization of circulation. The fetus was monitored at weekly intervals until the end of the 34th week of gestation. During the last fetal examination a IUGR of the fetus was confirmed with signs of starting centralization and anhydramnion. Subsequently, the patient was transferred to the delivery room where a positive oxytocin stress test was performed. In view of the finding a termination of pregnancy from the indication of lupus erythematosus systematicus gravis, hypoxio foetum intrauterina chronica, and IUGR was recommended.

A caesarean section was performed under general anesthesia. During intubation the tracheal mucosa was fragile, edematous and intubation was complicated with mild contact bleeding. A hypotrophic female newborn was born with a birth weight of $1300 \mathrm{~g}$, with an Apgar score of 2.6.8. Uterine hypotonia occurred after delivery of the placenta and umbilical cord. Despite the administration of Oxytocin and Methylergometrin the uterus was contracted only partially. During the procedure the uterine tissue and parametrial tissue were significantly fragile, soft, and edematous. During sewing the patient bleed from each puncture, the stitches were pruned. In an effort to stop the bleeding a bilateral uterine artery ligation was performed together with a left-side adnexectomy. During the surgery the following treatment was administrated: Exacyl 1g, Dicynone 250mg, Fibrinogen $2 g$, 3 transfusion units of erythrocytes, 3 frozen plasma units, Ca glucuronicum, glucocorticoids Dexona $4 \mathrm{mg}$, and ATB prophylaxis (Vulmizoline $1 \mathrm{~g}$ and Avrazor 500mg). Due to persistent severe bleeding without the tendency of blood clotting and total blood loss of approximatelly $1,600 \mathrm{ml}$ the surgeons decided to perform supravaginal hysterectomy in order to prevent development of disseminated intravascular coagulopathy. The surgical procedure was complicated by artificial perforation of the bladder apex. The urologist afterwards performed bilateral uretheral catheterization. The right ureter, despite the introduction of a well-maintained pig-tail drain, did not drain urine probably because of kidney insufficiency caused by SLE. After the surgery the patient was transported to the anesthesia ICU. After 24-hours monitoring and stabilization of vital signs the patient was transferred to the ICU of the Clinic of Internal Medicine. Lactation was stopped postoperatively. The postoperative course was without complications and the patient was released on the 9 th postoperative day to a multidisciplinary specialized outpatient care. Currently the patient is stabilized, on chronic HCQ and corticotherapy. She reagullary undergoes checks at a rheumatology department. The baby was released from the newborn department in a good physical state and still enjoys good health with no major health complications.

\section{DISCUSSION}

Systemic lupus erythematosus is a chronic inflammatory autoimmune disease with a high prevalence in women of childbearing age. The fact that chronic immunological diseases predominantly affect women suggests that female sex hormones play an important role in the pathophysiology of autoimmunity. Pregnancy, during which hormones are significantly elevated, is considered a high risk factor. Although women with SLE have on average fewer children than women in the general population, mainly due to increased abortion rates and lower birth rates, pregnancy is currently associated with significantly better outcomes in most patients with systemic lupus erythematosus than in the past (4). It is generally agreed that the risk of outbreak is determined by the activity of the disease before conception. A prospective observational study showed that patients with SLE disease activity index (SLEDAI) scores of 4 and more 6 months prior to pregnancy and with nephritis history had 
aggravated maternal outcomes (flares and preeclampsia), while lupus flares during pregnancy were a predictive factor for aggravated perinatal outcomes (5). Most outbreaks affect the mucocutaneous, musculoskeletal, and hematological systems. Outbreaks during pregnancy may be a key difference between uncomplicated pregnancy and maternal (lupus flare, deterioration of renal function, preeclampsia, and thrombotic events) or fetal-neonatal complications (abortion, preterm labor, intra-uterine lupus growth) (6).

When evaluating the disease activity during pregnancy it is difficult to distinguish the disease manifestations from physiological changes during pregnancy. Symptoms such as fatigue, mild thrombocytopenia, and anemia commonly encountered in pregnancy may be incorrectly assessed as evidence of increased disease activity. A very similar situation is with changes in complement concentrations that we consider classical markers of SLE activity. Therefore, the assessment of these biomarkers during pregnancy may not be credible (6). The distinction of whether or not a woman suffers from a lupus outbreak during pregnancy is often very difficult even for the most experienced professionals. It is also very difficult, but extremely important, to correctly distinguish preeclampsia from lupus nephritis (LN) whose manifestations are very similar (eg proteinuria, impaired renal function, or hypertension) but the treatment is different. The response to steroid therapy may be helpful (e.g. a rapid increase in platelet count for SLE). The presence of "nephritic" sediment with dysmorphic erythrocytes or cylinders is LN-specific (7). To distinguish physiological pregnancy changes from increased SLE activity it is necessary to look at the complete clinical picture (Table 1) (6).

Table 1 Symptoms that can help to distinguish SLE activity from physiological changes during pregnancy (6)

\begin{tabular}{|c|c|c|}
\hline & Pregnancy changes & SLE activity \\
\hline Clinical signs & $\begin{array}{l}\text { Fatique } \\
\text { Facial erythema } \\
\text { Palmar erythema } \\
\text { Arthralgia, myalgia } \\
\text { Mild swellings } \\
\text { Mild rest dyspnoe } \\
\text { Alopecia (post partum) }\end{array}$ & $\begin{array}{l}\text { Fever with no signs of infection } \\
\text { Lymfadenopathy } \\
\text { Photosensitive skin rush } \\
\text { Mouth and nasal mucosa ulceration } \\
\text { Inflamation arthritis } \\
\text { Serositis }\end{array}$ \\
\hline $\begin{array}{l}\text { Laboratory } \\
\text { parameters }\end{array}$ & $\begin{array}{l}\text { Mild anaemia } \\
\text { Mild trombocytopenia } \\
\text { Proteinuria }<300 \mathrm{mg} / \text { day } \\
\text { Elevation of complement factors }\end{array}$ & $\begin{array}{l}\text { Hemolytic anemia } \\
\text { Trombocytopenia } \\
\text { Leukopania, lymphopania } \\
\text { Proteinuria }>300 \mathrm{mg} / \text { day } \\
\text { Active urine sediment } \\
\text { Decrease of complement } \\
\text { Increase of dsDNA antibodies }\end{array}$ \\
\hline
\end{tabular}

\section{Treatment of SLE during pregnancy}

An important aspect in pregnancy management is the optimization of drug treatment before and during this period. As the risk of outbreaks is increased during or after pregnancy the patients with SLE should continue to receive medicines that can be taken during pregnancy. However, patients but sometimes even treating doctors, often discontinue treatment because of toxicity concerns, leading to outbreaks of avoidable illness and the associated complications. Therefore, it is extremely important to inform patients not only about the risks of drug treatment but also about the risks of under-treated disease. EULAR has 
recently produced an overview of the safety of antirheumatic drugs before, during, and after pregnancy and, in addition to the 4 basic principles, it has made recommendations that should be taken into account when prescribing individual medicines (8). A summary of the safety of each drug is shown in Table 2 .

Table 2 Drug compatibility during pregnancy and lactation (8)

\begin{tabular}{|c|c|c|}
\hline & Allowed in pregnancy & Allowed during lactation \\
\hline Hydroxychlorochin & Yes & Yes \\
\hline Azathioprine & Yes & Yes \\
\hline $\begin{array}{l}\text { Neflorinovan } \\
\text { glucocorticoids } \\
\text { (prednisone a prednisolone) }\end{array}$ & Yes & Yes \\
\hline $\begin{array}{l}\text { Florinated glucocorticoids } \\
\text { (dexametasone } \\
\text { a betametasone) }\end{array}$ & $\begin{array}{l}\text { Yes, it should be limited to situations } \\
\text { where } \\
\text { fetal treatment is required }\end{array}$ & Yes \\
\hline Ciclosporine & Yes & Yes \\
\hline Tacrolimus & Yes & Yes \\
\hline $\begin{array}{l}\text { Intravenous } \\
\text { immunoglobuline }\end{array}$ & Yes & Yes \\
\hline Methotrexate & No, stop 3 months before conception & No (insufficient data) \\
\hline Mycophenolate mofetil & $\begin{array}{l}\text { No, stop at least } 6 \text { weeks before con- } \\
\text { ception }\end{array}$ & No available data \\
\hline Cyclophosphamid & $\begin{array}{l}\text { No. Stop at least } 3 \text { months before con- } \\
\text { ception }\end{array}$ & No available data \\
\hline Belimumab & $\begin{array}{l}\text { No (insufficient data) Unplanned expo- } \\
\text { sure in the first trimester is low risk }\end{array}$ & No available data \\
\hline Rituximab & $\begin{array}{l}\text { No (insufficient data) Unplanned expo- } \\
\text { sure in the first trimester is low risk }\end{array}$ & No available data \\
\hline ACE inhibitors & No, stop when a pregnancy is confirmed & Yes (limited data) \\
\hline NSAIDs & Yes, stop before 32nd week & Yes \\
\hline
\end{tabular}

NSAIDs are well tolerated drugs in the first two trimesters of pregnancy. Their use should be discontinued after 30 weeks of gestation as they may cause premature closure of the ductus arteriosus. There is no data on selective COX-2 inhibitors, therefore it is recommended that treatment be changed to conventional NSAIDs if possible. Unflorinated glucocorticoids, prednisone and prednisolone, are considered first-line medicines for lupus flares and can be used during pregnancy. Only minimal amounts of these glucocorticoids reach the fetus through the placenta, particularly due to significant placental metabolism. However, the daily dosage should be adapted to the disease activity and the duration of treatment should be minimized as much as possible (ideally $7.5 \mathrm{mg}$ prednisolone per day). Taking 
higher doses (more than $10 \mathrm{mg} /$ day) for several weeks increases the risk of adverse effects (gestational diabetes, hypertension, IUGR, preeclampsia, and premature labor). A recent study found that there was a significantly increased risk of serious infection in pregnant women with systemic inflammatory disease (9).

The use of florinated compounds such as dexamethasone and betamethasone should be limited to situations where treatment of the fetus is necessary (e.g. promoting lung maturation or severe forms of congenital heart block). HCQ, azathioprine, cyclosporine, tacrolimus, and intravenous globulins are considered to be safe during pregnancy. HCQ can offer several benefits not only to patients with mild SLE but also brings significant benefits to patients with multiple organ systems disfunction. Its positive effect on reducing the risk of flare-ups, obstetric complications, thrombosis, and congenital heart block has been proven. Several mechanisms are known to work with antimalarial drugs which are very similar to those that play a role in preeclampsia (10).

Cyclophosphamide, Mycophenolate mofetil and methotrexate are teratogenic substances and their use must be discontinued before pregnancy. The use of biological therapy during pregnancy is gradually becoming more common but there is still a lack of data on antiTNF active substances that are not a typical therapeutic option for SLE. Data on the use of other biological agents, e.g. rituximab and belimumab, are obtained from case studies or safety registries from the manufacturers themselves but indicate a high level of "undetected" risks. Assessing their risks in connection with pregnancy is currently not possible. However, the data obtained so far have not yielded any signals of an increased rate of malformations. Therefore, they should only be used during pregnancy when other pregnancy-compatible medicines cannot be used in the treatment of SLE (11).

Any woman planning pregnancy should take $400 \mathrm{mg}$ of folic acid daily, which significantly reduces the risk of developing neural tube defects in the fetus, ideally at least 3 months before pregnancy and during the first trimester. If the patient has been taking methotrexate in the previous 3 months, she should increase the daily dose up to $5 \mathrm{mg}$ of folic acid per day. All pregnant SLE patients, especially those taking glucocorticoids and LMWH, should receive 1,000 IU of vitamin D until the end of lactation. Lupus patients are at a higher risk of vitamin D deficiency and may require higher doses. Calcium is also recommended not only in women on corticosteroid and LMWH treatment as there is an increased risk of osteoporosis. A meta-analysis showed that a daily minimum dose of $1 \mathrm{~g}$ calcium (vs placebo) resulted in a $50 \%$ reduction in the risk of developing pre-eclampsia and a $25 \%$ reduction in the risk of premature labor. Patients with lupus and renal impairment, hypertension, and previous preeclampsia are also recommended to start a low-dose aspirin therapy in early pregnancy (12).

\section{Extracorporeal treatment methods}

Extracorporeal treatments such as exchange plasmapheresis and immunoadsorption are used in the treatment of multiple autoimmune disorders such as systemic lupus erythematosus, myasthenia gravida, idiopathic dilated cardiomyopathy, glomerular disorders (eg, ANCA associated glandular vasculitis ) and in patients undergoing desensitization prior to kidney transplantation (13).

\section{PLASMAPHERESIS}

Plasmapheresis is an extracorporeal blood purification method designed to remove high molecular weight substances from plasma. Therapeutic exchange plasmapheresis is performed when harmful substances (autoantibodies, immunocomplexes, monoclonal proteins, cryoglobulins, plasma protein-bound toxins) are present in the plasma or the physiological parts of the plasma are over-multiplied. This treatment process has been used to treat multiple autoimmune diseases including systemic lupus erythematosus since the 1970s. A more specific form of extracorporeal therapy is immunoadsorption which, like PLEX, has a positive effect on patients with refractory disease with contraindications to standard immunosuppressive therapy or during pregnancy (14). 
Exchange plasmapheresis is associated with a decrease in serum immunoglobulins of almost $60 \%$ while using new devices after 2-4 cycles of therapy and may result in similar decreases in circulating immunocomplexes, ANA, anti-dsDNA, and antiphospholipid antibodies. Adjustment of complement component levels and the increase in erythrocyte receptor type 1 complement have also been reported. Effectiveness of PLEX is confirmed also by the increase in interferon- positive T-helper cells and CD4 + CD25 (high) FoxP3 + T-cells and decrease in IL-4 and IL-10 producing CD4 + T-cells. Extracorporeal therapy also leads to a decrease in SCD40L, a marker of the active form of the disease (15).

The studies published so far are difficult to interpret reliably due to their design and low patient numbers but the benefit of PLEX has been mainly described in patients with refractory severe disease, during pregnancy, or in patients with antiphospholipid syndrome and in women who had pregnancy complications in the past. In assessing the effectiveness of PLEX, consideration should also be given to the effects of concomitant therapy, the correct distinction between the symptoms of reversible and irreversible forms of SLE, the different PLEX dosing rates, and the inclusion of patients with newly diagnosed SLE who tend to respond well to standard therapy. PLEX can be used as a treatment method in patients with refractory lupus nephritis or central nervous system disease or SLE-associated haematological conditions. In a small multicenter study comparing PLEX in combination with steroids with cytotoxic drugs and steroids, Derksen and his team did not find a difference in kidney impact results. Therefore, if the patient is at high risk of developing a serious infection, PLEX can be used as a therapeutic method (16).

However, the optimal duration of PLEX has not been established and it is necessary to define the extent of treatment in individual patients. It is also worth remembering that during PLEX it is necessary to provide alternative therapy to maintain oncotic pressure. In this case 5\% albumin or freshly frozen plasma is possible. While $5 \%$ albumin has the advantage of viral inactivation, it is stored at room temperature and is associated with minimal adverse effects and has iso-oncotic properties, fresh frozen plasma has more physiological properties as it replaces all plasma components. At the same time, however, it is associated with several disadvantages such as the risk of transmission of viral particles, difficult storage, blood group compatibility, the risk of an allergic reaction, and lack of availability (17).

\section{IMMUNOABSORPTION THERAPY}

Also, extracorporeal treatment with IAS can be considered an alternative in the treatment of severe manifestations of SLE, especially in those patients who do not respond to traditional treatment or with contraindications to traditional treatment. The first mention of IAS in the treatment of SLE was published in 1990 and interest in this form of treatment has steadily increased since then (18).

Several IAS columns are used to treat patients with SLE. Thanks to IAS columns with high afinity it is possible to remove lupus-specific antibodies such as anti-dsDNA antibodies during one cycle of therapy. Total serum IgG decreases by $75 \%$. This efficiency even surpasses the ability of sequential PLEX therapy. However, it should be remembered that decreasing the amount of immunoglobulins below 3g/1 may increase the risk of serious infections in patients on concomitant cytotoxic therapy and high dose steroid therapy (19). Low affinity adsorption columns have a lower efficiency. Some specific columns can remove disease-specific antibodies but have not been shown to lead to C3/C4 complement elevation. Complement adjustment and decrease of anti-dsDNA antibodies can be achieved in most patients using other columns. Treatment with high affinity columns resulted in a reduction in GM-CSF, IL-10 and CD4 + / CD71 + T cells, whereas a removal of anaphylatoxins $\mathrm{C} 3 \mathrm{a}$ and $\mathrm{C} 4 \mathrm{a}$ was observed in patients treated with low affinity IAS columns. A decrease in anti-VRT-101 antibodies was observed after using some specific columns (20).

Similarly to PLEX efficacy studies there are several differences in the IAS studies that limit the validity of their results. However, several studies describe the positive effect of IAS in 
the treatment of pregnant patients with SLE, which may be one of the important indication criteria when using this extracorporeal treatment method. However, it remains to determine an optimal duration of treatment along with the optimization of plasma processing (21).

\section{REFERENCES}

1. Kronbichler A, Mayer G. Renal involvement in autoimmune connective tissue diseases. BMC Med 2013;11:95.

2. Bertsias GK, Tektonidou M, Amoura Z, Aringer M, Bajema I, Berden JH, et al. Joint European League Against Rheumatism and European Renal Association-European Dialysis and Transplant Association (EULAR/ERA-EDTA) recommendations for the management of adult and paediatric lupus nephritis. Ann Rheum Dis 2012;71: 1771-82.

3. Mulhearn B, Bruce IN. Indications for IVIG in rheumatic diseases. Rheumatology 2015;54:383-91.

4. Clowse ME, Chakravarty E, Costenbader KH, et al. Effects of infertility, pregnancy loss, and patient concerns on family size of women with rheumatoid arthritis and systemic lupus erythematosus. Arthr Care Res 2012; 64(5): 668e74.

5. Buyon JP, Kim MY, Guerra MM, et al. Kidney outcomes and risk factors for nephritis (Flare/De Novo) in a Multiethnic cohort of pregnant patients with lupus. Clin J Am Soc Nephrol 2017; 12(6): $940 \mathrm{e} 6$

6. Buyon JP, Cronstein BN, Morris M, et al. Serum complement values (C3 and C4) to differentiate between systemic lupus activity and preeclampsia. Am J Med 1986; 81(2): 194e200.

7. Jakobsen IM, Helmig RB, Stengaard-Pedersen K. Maternal and foetal outcomes in pregnant systemic lupus erythematosus patients: an incident cohort from a stable referral population followed during 1990-2010. Scand J Rheumatol 2015; 44(5): 377e84.

8. Gotestam Skorpen C, Hoeltzenbein M, Tincani A, et al. The EULAR points to consider for use of antirheumatic drugs before pregnancy, and during pregnancy and lactation. Ann Rheum Dis 2016; 75(5): 795e810.

9. Andreoli L, Bertsias GK, Agmon-Levin N, et al. EULAR recommendations for women's health and the management of family planning, assisted reproduction, pregnancy and menopause in patients with systemic lupus erythematosus and/or antiphospholipid syndrome. Ann Rheum Dis 2017; 76(3): 476e85.

10. Abd Rahman R, DeKoninck P, Murthi P, et al. Treatment of preeclampsia with hydroxychloroquine: a review. J Matern Fetal Neonatal Med 2017: 1e5. https://doi.org/10.1080/14767058.2017. 1289511 [Epub ahead of print].

11. Flint J, Panchal S, Hurrell A, et al. BSR and BHPR guideline on prescribing drugs in pregnancy and breastfeeding-Part I: standard and biologic disease modifying anti-rheumatic drugs and corticosteroids. Rheumatol Oxf 2016; 55(9): 1693e7.

12. Hofmeyr GJ, Lawrie TA, Atallah AN, et al. Calcium supplementation during pregnancy for preventing hypertensive disorders and related problems. Cochran Database Syst Rev 2014;(6),CD001059.

13. Muangchan C, van Vollenhoven RF, Bernatsky SR, Smith CD, Hudson M, Inanc M, et al. Treatment algorithms in systemic lupus erythematosus. Arthritis Care Res Mar 162015. http://dx.doi.org/ 10.1002/acr.22589 [epub ahead of print].

14. Andreas Kronbichler, Biljana Brezina, Luis F. Quintana, David R.W. Jayne. Efficacy of plasma exchange and immunoadsorption in systemic lupus erythematosus and antiphospholipid syndrome: A systematic review. Autoimmunity Reviews 15 (2016) 38-49

15. Zhang YY, Tang Z, Chen DM, Gong DH, Ji DX, Liu ZH. Comparison of double filtration plasmapheresis with immunoadsorption therapy in patients with anti-glomerular basement membrane nephritis. BMC Nephrol 2014;15:128.

16. Derksen RH, Hene RJ, Kallenberg CG, Valentijn RM, Kater L. Prospective multicentre trial on the short-term effects of plasma exchange versus cytotoxic drugs in steroidresistant lupus nephritis. Neth J Med 1988;33:168-77. 
17. McLeod BC. Plasma and plasma derivatives in therapeutic plasmapheresis. Transfusion 2012;52(Suppl. 1):38S-44S

18. Schneider M, Berning T, Waldendorf M, Glaser J, Gerlach U. Immunoadsorbent plasma perfusion in patients with systemic lupus erythematosus. J Rheumatol 1990; 17:900-7.

19. Schwenger V, Morath C. Immunoadsorption in nephrology and kidney transplantation. Nephrol Dial Transplant 2010;25:2407-13.

20. Stummvoll GH, Schmaldienst S, Smolen JS, Derfler K, Biesenbach P. Lupus nephritis:prolonged immunoadsorption (IAS) reduces proteinuria and stabilizes global diseaseactivity. Nephrol Dial Transplant 2012;27:618-26.

21. Bartošová L, Fedorová J, Ivanková J, Hollý P et al. Sticky platelet syndrome: Where is its cause? Acta Medica Martiniana 2009;3:23-29.

\section{List of abbreviations}

ACE - angiotensine covertine enzyme

ANA - anti nuclear antibody SLEDAI- systemic lupus erythematosus disease activity index

ANCA - Anti-neutrophil cytoplasmic antibody

ASA - acetylsalicylic acid

COX - cyclooxygenase

EULAR - The European League Against Rheumatism

HCN - hydrocortison

HCQ - hydroxychloroquine

IAS - immunoadsorption

ICU - intensive care unit

IUGR - intrauterine growth restriction

LN - lupus nephritis

LMWH - low molecular weight heparine

NSAIDs - Nonsteroidal anti-inflammatory drugs

PLEX - plasmapheresis / plasma Exchange

SLE - Systemic lupus erythematosus

Received: February, 11, 2020

Accepted: May, 25, 2020 